FEATURE ARTICLE

\section{Nanotechnology in medicine}

\author{
D. Nedra Karunaratne \\ Department of Chemistry, University of Peradeniya, Peradeniya.
}

Submitted: 23 May 2006 ; Accepted: 15 June 2007

\section{INTRODUCTION}

In the past few years, the interest in nanoscale science or nanoscience has shown an exponential increase. Nanoscience relates to the study of properties, phenomena and the response of materials at the submicron range. Below a certain length scale, a system behaves differently; and the ability to predict that behavior by extrapolating large to small is lost. In the nanoscale, properties of matter have been found to be very different from those of micro or macroscale. Quantum effects play a very important role in the nano range due to the high surface to volume ratio when compared to large particles. The applications of nanoscience nanotechnology, has enormous potential in information and communication technology, biology and medicine. Since most of these applications are centred on improving human health and diagnosing the status of human well being, nanobiotechnology has given rise to a whole new field: nanomedicine - the medicine of tomorrow.

Nanomedicine encompasses science and technology which uses molecular tools and knowledge of the human body for :

- producing novel therapeutics (new methods of curing diseases and tissue repair)

- developing diagnostics for rapid monitoring (high throughput arrays, imaging devices, nanoparticle labels, ultra sensitive detectors or nanobiosensors)

- developing biopaharmaceutics (targeted drug delivery and gene delivery systems)

- improving cell- material interactions (implantable materials and scaffolds for tissue repair, cochlear implants and retinal implants)

- developing surgical tools and surgical aids (operating tools and surgical robots)
With the discovery of the DNA double helix in the mid $20^{\text {th }}$ century, molecular biology became the emerging science by the end of the century. The marriage of biotechnology with nanotechnology opened wider horizons and the quest for intelligent nanodevices gave new life to development in the area of nanomedicine. Advanced diagnostics, nanobiosensors, smart drugs and delivery systems are all a result of these improved molecular technologies ${ }^{1}$. To delve further into the future, possibilities of developing computer controlled artificial nanostructures which are smaller than the cell are currently being looked into. This feature article presents a general overview of the important applications of nanotechnology in medicine ${ }^{2}$.

\section{Applications in drug delivery}

Drugs may be delivered to the human body by oral methods, through contact, via the respiratory system, through parental administration or by implants. All these methods require the drug being delivered to be in a form that is easily and rapidly absorbed into the system for fast action at the target site. Some drugs or therapeutic agents, which show promise in laboratory trials, fail to produce the same effect in the human body. This may be due to degradation of drug before it reaches the target site (poor bioavailability or inability to cross the blood brain barrier) or poor solubility of the drug in the delivery medium, resulting in administration of high doses to the patient leading to severe side effects.

Intelligent material design at the nanoscale has given us nanomaterials which can be utilized as nanocarriers for efficient drug delivery ${ }^{3}$. The main advantages of nanocarriers over other drug carriers are:

a) their small size, which helps them overcome physiological barriers and gain entry into cells. 
b) increased solubility improves its bioavailability.

c) ability to be delivered to specific target sites with controlled release by specific signals based on temperature sensitivity or magnetic property.

d) efficient delivery of proteins, nucleic acids and other small molecules.

Nanocarriers have the added advantage of being able to penetrate tumours and can be used as targeted delivery systems in cancer treatment with reduced toxicity and side effects. They can be made biocompatible by attaching a biological coating or a layer which can act as an interface onto the material.

Nanocarriers are smaller than $1 \mu \mathrm{m}$ (submicronic) and can take many forms ${ }^{4}$ such as liposomes, nanosuspensions, nanospheres (passive colloidal carriers), nanocapsules, nanotubes, nanowires and dendrimers. Drugs (especially proteins and peptides) included within liposomes have altered pharmacokinetic profiles. Polymeric matrix systems in which the drug is uniformly dispersed are nanospheres, while a nanocapsule encapsulates or surrounds the drug dissolved or entrapped in an oil or aqueous core. The matrix system is a biodegradable and biocompatible synthetic or natural polymer. Natural polymer nanoparticles are superior at delivering drugs, are biodegradable, have reduced side effects, increased absorption, and prolonged therapeutic effects when compared to synthetic polymer matrices:

Sheets of carbon atoms arranged in the form of tubes (diameter 1-20 $\mathrm{nm}$ ) are called nanotubes and can deliver DNA. proteins and peptides. A dendrimer is a spherical macromolecule with diameter of 1-10 nm having a series of branches around an inner core. Drugs, markers or receptors can be attached to the many branches on a dendrimer making it not only a drug carrier, but a multifunctional probe. This has been successfully demonstrated by attaching methotrexate (anticancer drug), folic acid (as a targeting agent towards cancer cells) and a fluorescent imaging agent (to monitor the drug delivery) to a dendrimer 5 .

Nanocarriers may be made more versatile by attaching functional groups. Functionalization enables their use in biosensing, bio imaging, as cell markers, as monitors of antigen antibody reactions and as vaccines in addition to drug delivery. The targeting potential and circulation time is also increased.

\section{Applications in molecular imaging}

In cancer therapy, the shrinkage of a tumour in response to treatment, or pathological changes in an organ is measured at the macro level by conventional imaging methods. In contrast, molecular imaging deals with diagnosing the onset of a disease at the molecular level, prior to clinical onset or occurrence of symptoms. An imaging molecule coupled to a nanoparticle can be functionalized to target receptors or ligands specific to a disease. Since these markers are present only on the diseased tissues, accumulation of the contrast medium within, allows diagnosis prior to the development of symptoms.

One of the more commonly used molecular imaging tools is quantum dots (QD's). Unlike fluorescent dyes which are large, QD's are nanosized, non toxic and are stable to photobleaching. Several uses of QD attached probes for tumour detection have been reported $\mathrm{d}^{\text {. }}$.

Super paramagnetic iron oxide nanoparticles (SPION) are small synthetic $v \mathrm{Fe}_{2} \mathrm{O}_{3}$ or $\mathrm{Fe}_{3} \mathrm{O}_{4}$ particles with a core size of $<10 \mathrm{~nm}$ and an organic or inorganic coating. SPION coated with dextran have been in use in Europe for the past 10 years as contrast agents for Magnetic Resonance Imaging (MRI) of the liver. These nanoparticles can increase the diagnostic sensitivity and specificity by modifying the relaxation time of protons.

\section{Applications in diagnostics}

Diagnostics depend on the analysis of a response and the efficiency of the detector system. Biosensors are analytical devices which convert a biological response into a detectable signal. The bioreceptor, which binds or interacts with the analyte in a biosensor may be categorized depending on the 1ype of biological response. Five categories are recognized:

i. An antigen-antibody based bioreceptor senses the binding event between an antibody and antigen.

ii. Enzyme based bioreceptors can detect the results of an enzyme catalyzed reaction.

iii. Nucleic acid interactions with proteins, enzymes or DNA are detected by DNA biosensors, genosensors or biodetectors. Micro electro mechanical systems (MEMS) and nano electro mechanical systems (NEMS) may be used to perform polymerase chain reactions to increase the DNA concentration to detectable limits. DNA encoded nanoparticle probes unique to a target protein of interest has been used to detect biomarkers in Alzheimer's disease ${ }^{7}$.

iv. Cellular interaction based biosensors detect global parameters. For example, genetically engineered bacteria may detect chemical agents, toxic metal ions, DNA damaging agents or even stress conditions. 
iv. Interaction with synthetic materials. - Aptamers are biomimetic materials like artificial nucleic acid ligands or peptide domains on a protein scaffold which can recognize and bind specific targets. Vascular endothelial growth factor (VEGF) is a signaling protein that has been used as a serum biomarker for rheumatoid arthritis, breast cancer, lung cancer, and colorectal cancer and is also associated with agerelated macular degeneration (AMD). The first FDA approved aptamer based drug in treatment for AMD, called Macugen is offered by OSI pharmaceuticals.

Biosensors are very versatile in diagnostics. There are several applications such as Lab-on-a-chip devices, micro arrays for diagnostics and microfluidic devices. These medical devices are for in vitro analysis and hence do not cause safety concerns unlike invasive techniques where nanoparticles are introduced into the body.

\section{Applications in cardiology}

The applications of nanotechnology in cardiology and vascular processes may be diagnostic or therapeutic. Cardiovascular diseases may be diagnosed by using biospecific antibodies tagged with radio labeled nanopolymers to target atherosclerotic lesions for detection by imaging. Biosensors employing carbon nanotube - DNA interactions may be produced to identify multiple genes associated with coronary artery disease (CAD). On the therapeutic side, stent implantation was developed to overcome the acute recoil and high restinosis rate of balloon angioplasty. Although recent studies have shown that drug-eluting stents (DES) are associated with increased risk of late-stent thrombosis, DES have emerged as one of the most promising technologies in the field of interventional cardiology ${ }^{8}$. Currently, sirolimus, paclitaxel and zotarolimus eluting stents are among the most popular DES available commercially. Targeted nanoparticles could help ensure that anti restenotic drugs are available for all lesions ${ }^{9}$ while the removal of anti proliferative agents from the intimal surface into the vascular wall may help enhance rehealing of the endothelium, improving post procedural management of these patients.

\section{Tissue engineering}

Creation of artificial cells, tissues and organs is another area where nanotechnology can be applied ${ }^{10}$. Polymeric scaffolds for tissue engineering can be prepared by many different techniques. Weigel et al. reviews methods such as electrospinning, induced phase separation of polymer solutions or porogen leaching, usage of self assembly systems that mimic the properties of the extra cellular matrix (ECM) and novel fabrication techniques such as rapid prototyping or solid free form procedures ${ }^{13}$. Electrospinning can produce a macroporous scaffold comprising randomly oriented nanofibres which can accommodate drug delivery function as well as its use in tissue engineering ${ }^{12}$.

\section{Applications of nano devices}

R. A. Freitas Jr. has predicted the use of nanorobots and nanodevices for medical applications. The "respirocyte" is a $1 \mu \mathrm{m}$ diameter sphere mimicking a red blood cell ${ }^{13}$ with ability to deliver more oxygen to the tissue than natural red blood cells. The "microbivore" is the artificial version of a white blood cell ${ }^{14}$. Freitas' newest futuristic idea is a self powered, computer controlled medical nanorobot - a "pharmacyte" with ability of controlled drug delivery to specific targets at specified times ${ }^{15}$ and may be used for initiation of apoptosis in cancer cells and direct control of cell signaling processes.

Artificial red blood cells resembling natural RBC's constructed by Chang ${ }^{16}$ are nanocapsules containing hemoglobin and enzymes in a polylactic glycolic acid as matrix. These artificial RBC's have a circulation time nearly double that of polyhemoglobin blood substitutes.

\section{Applications in cancer therapy}

New developments in cancer therapeutics and drug delivery have shown tremendous advancement in recent years. However, general treatment of cancers in patients may not have the same results due to variations in individuals and variations in the behaviour of cancers of the same histological type from one patient to another ${ }^{17}$. By understanding the molecular mechanisms of cancers and improving molecular diagnostics, a personalized approach to management and treatment will be possible. Nanobiotechnology has the potential to improve early detection of cancer biomarkers through nanobiosensors and nanoimaging techniques leading to nanodiagnostic techniques. Application of proteomic technology to cancer detection can take the form of nanoscale protein analysis as a replacement to biopsies. Oncoproteomics (application of protein technology to oncology) may be utilized to design drugs according to the molecular profile of the cancer cell. By combining these diagnostics with therapeutics and drug delivery systems, management of cancer patients with personalized medicine is envisaged.

\section{Nanomedicine in the future}

The National Institute of Health (NIH) and the National Cancer Institute of the United States have begun 
programmes (NIH Roadmap of Nanomedicine Initiatives) to develop nanoscale technologies such as nanodiagnostics, microchip devices, drug delivery agents and cancer diagnostics and therapeutics ${ }^{18}$. Within the next 10 years they hope to begin harvesting the medical benefits of these programmes. The government of Sri Lanka also plans to introduce Nanotechnology to industrialists ${ }^{19}$.

\section{References}

1. I.ogothetidis S. (2006). Nanotechnology in Medicine: the medicine of tomorrow and nanomedicine. Hippokratia 10: $?-21$.

2. Kubic T., Bogunia-Kubik K. \& Sugisaka M. (2005). Nanotechnology on duty in medical applications. Current Pharmaceutical Biotechnology 6: $17-33$.

3. Rawat M., Singh D., Saraf S. \& Saraf Swarnlaata (2006). Nanocarriers: promising vehicle for bioactive drugs. Biological and Pharmaceutical Bulletin 29: 1790-1798.

4. Koo O.M., Rubinstein I. \& Onyuksel H. (2005). Role of nanotechnology in targeted drug delivery and imaging: a concise review. Nanomedicine 1: 193-212.

5. Kukowska-Latallo J.F., Candido K.A., Cao Z., Nigavekar S.S., Majoros I.J., Thomas T.P., Balogh L.P., Khan M.K. \& Baker J.R. Jr. (2005). Nanoparticle targeting of anticancer drug improves therapeutic response in animal model of human epithelial cancer. Cancer Research 65: 5317-5324.

6. Wang Y., Tang Z. \& Kotov N.A. (2005). Bioapplication of nano semiconductors. Nanotoday $20-30$.

7. Georganopoulou D.G., Chang L., Nam J. M., Thaxton C. S., Mufson E.J., Klein W.L. \& Mirkin C.A. (2005). Nanoparticle-based detection in cerebral spinal fluid of a soluble pathogenic biomarker for Alzheimer's disease. Proceedings of the National Academy of Science USA. 102: $2263-2264$.
8. Garcia-Garcia H.M., Vaina S., Tsuchida K. \& Serruys P.W. (2006). Drug- eluting stents. Archivos de Cardiologia de Mexico 76: $297-319$.

9. Lanza G., Winter P., Cyrus T., Caruthers S., Marsh J., Hughes M. \& Wickline S. (2006). Nanomedicine opportunities in cardiology. Annals of the New York Academy of Sciences 1080: 451 - 465.

10. Kubik T., Bogunia-Kubik K. \& Sugisaka M. (2005). Nanotechnology on duty in medical applications. Current Pharmaceutical Biotechnology 6: 17 - 33.

11. Weigel T., Schinkel G. \& Lendlein A. (2006). Design and preparation of polymeric scaffolds for tissue engineering. Expert Review of Medical Devices 3: 835 - 851.

12. Chew S.Y., Wen Y., Dzenis Y. \& Leong K.W. (2006). The role of electrospinning in the emerging field of nanomedicine. Current Pharmaceutical Design 12: 4751 -4770 .

13. Freitas R.A. Jr. (1998). Exploratory design in medical nanotechnology: a mechanical artificial red cell. Artificial Cells, Blood Substitutes and Immobilization Biotechnology. 26: $411-30$.

14. Freitas R.A. Jr. (2005). Microbivores: artificial mechanical phagocytes using digest and discharge protocol. Journal of Evolution and Technology 14: 55 - 105.

15. Freitas R.A. Jr. (2006). Pharmacytes: an ideal vehicle for targeted drug delivery. Journal of Nanoscience and Nanotechnology 6: $2769-2775$.

16. Chang T.M. (2003). Future generations of red blood cell substitutes. Journal of Internal Medicine 253: 527 - 535.

17. Jain K.K. (2005). Role of nanobiotechnology in developing personalized medicine for cancer. Technology in Cancer Research and Treatment 4: $645-650$.

18. Moghimi S.M., Hunter A.C. \& Murray J.C. (2005). Nanomedicine: current status and future prospects. Federation of American Societies for Experimental Biology (FASEB) Journal 19: 311 -- 330.

19. http://www.asiantribune.com/index.php?q=node $/ 2483$. 